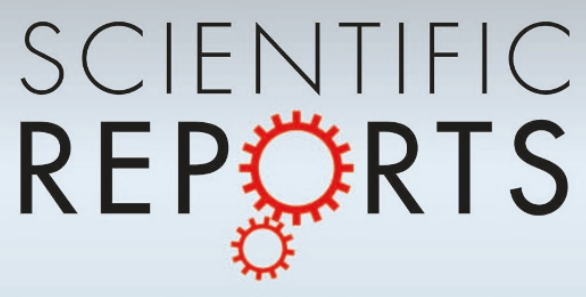

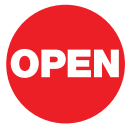

SUBJECT AREAS:

ELECTRONIC MATERIALS

AND DEVICES

APPLIED PHYSICS

IMAGING

MODELLING AND THEORY

Received

25 June 2012

Accepted

30 July 2012

Published

17 August 2012

Correspondence and requests for materials should be addressed to

K.Y.Z. (mpezk@nus. edu.sg) or J.D. (msedingj@nus.edu.

sg)

* These authors contributed equally to this work.

\title{
Investigation of the non-volatile resistance change in noncentrosymmetric compounds
}

\author{
T. S. Herng ${ }^{1 *}$, A. Kumar ${ }^{2 *}$, C. S. Ong' ${ }^{1}$, Y. P. Feng ${ }^{3}$, Y. H. Lu' ${ }^{4}$ K. Y. Zeng ${ }^{2} \&$ J. Ding'
}

\begin{abstract}
'Department of Materials Science and Engineering, National University of Singapore, 9 Engineering Drive 1, Singapore 117576 , Singapore, ${ }^{2}$ Department of Mechanical Engineering, National University of Singapore, 9 Engineering Drive 1, Singapore 117576 , Singapore, ${ }^{3}$ Department of Physics, National University of Singapore, 2 Science Drive 3, Singapore 117542, Singapore,

${ }^{4}$ Department of Materials Science and Engineering, Zhejiang University, Hangzhou 310027, China.
\end{abstract}

Coexistence of polarization and resistance-switching characteristics in single compounds has been long inspired scientific and technological interests. Here, we report the non-volatile resistance change in noncentrosymmetric compounds investigated by using defect nanotechnology and contact engineering. Using a noncentrosymmetric material of $\mathrm{ZnO}$ as example, we first transformed $\mathrm{ZnO}$ into high resistance state. Then $\mathrm{ZnO}$ electrical polarization was probed and its domains polarized $180^{\circ}$ along the [001]-axis with long-lasting memory effect ( $>25$ hours). Based on our experimental observations, we have developed a vacancy-mediated pseudoferroelectricity model. Our first-principle calculations propose that vacancy defects initiate a spontaneous inverted domains nucleation at grain boundaries, and then they grow in the presence of an electrical field. The propagation of inverted domains follows the scanning tip motion under applied electrical field, leading to the growth of polarized domains over large areas.

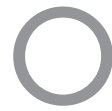

$\mathrm{n}$ the road towards higher memory density and computer performance, a significant improvement in energy efficiency constitutes the dominant goal in future materials studies. The materials with resistance switching ${ }^{1}$ behavior or ferroelectricity ${ }^{2}$ have been widely studied for their nonvolatile memory applications. The resistance switching (Fig. 1b) signifies a dramatic change in resistance under current-limited electrical field modulation ${ }^{1}$. The system in low resistance state (LRS) is switched by the threshold voltage into high resistance state (HRS) and vice versa, elucidating the properties of resistance random access memory (RRAM). Many oxide materials, including $\mathrm{ZnO}^{3}$, have shown resistance switching behavior. Possible explanations for the switching behavior include oxygen ion/vacancy migration ${ }^{4}$ and formation/annihilation at the metal/ oxide interface ${ }^{5}$, anion migration-induced redox switching ${ }^{1}$, phase changes in thin film ${ }^{6}$, and the electron-spin degree of freedom for semiconductor/half metal junctions ${ }^{7}$.

On the other hand, ferroelectricity is a cooperative phenomenon that enables the electrical polarization switching (up/down) under an applied electrical field with a negligible current flow. Interestingly, there are number of materials (e.g. $\mathrm{ZnO}, \mathrm{GaN}, \mathrm{BeO}$, etc) appear naturally as noncentrosymmetric polar structure. These materials consist of bilayers of alternating positively and negatively charged ions, leading to a spontaneous polarization theoretically ${ }^{8,9}$. Due to inevitable existence of intrinsic defects and intentional doping, it is essential to take doping effects into consideration when studying spontaneous polarization in such materials. Several experiments have been designed to probe the polarization switching and ferroelectric-like behavior in doped $\mathrm{ZnO}$ and $\mathrm{GaN}^{10-13}$. Tagantesev proposed that the ferroelectric-like phenomena in doped $\mathrm{ZnO}$ are compatible with Landau model and it is classified as "pseudoferroelectric" or "pseudoferroelectricity"14. However, the physical origin of the ferroelectric-like behavior in noncentrosymmetric compound is under vociferous debate. One of the rankling obstacles is the insufficient insulating property in these noncentrosymmetric compounds which has hindered the ferroelectric measurement because the applied electric field is screened by conduction electrons. In addition, physical fundamental of polarization switching and/or the formation of inverse ferroelectric domain / domain propagation need to be established.

Tapping into the intriguing properties of polarization and resistance switching in noncentrosymmetric compounds, it is technologically desirable to develop a single material that coexistence of resistance and polarization switching properties. With such materials system, one can probe its polarization switching behavior, when the sample is in the high resistance regime. Here, we demonstrate the hybrid switching (polarization and resistance 


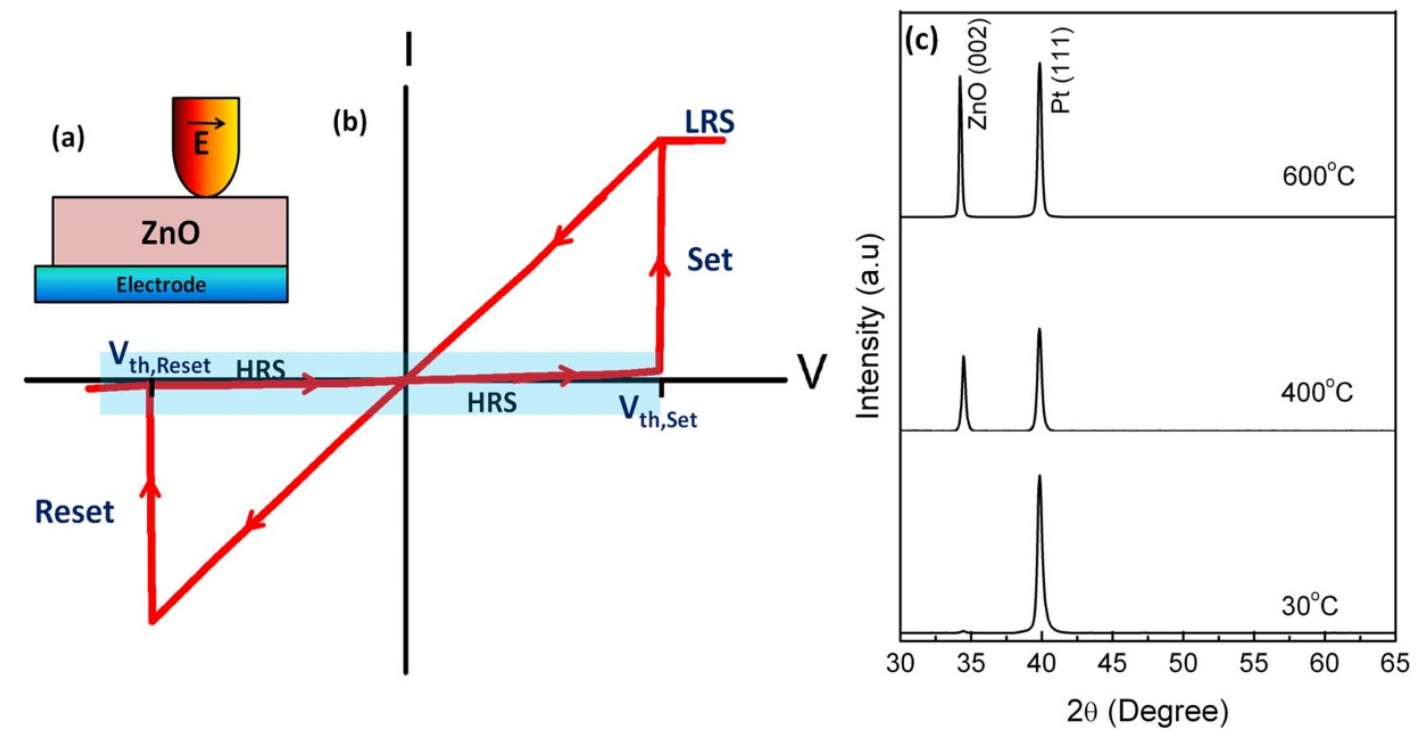

Figure $1 \mid$ The conceptual resistance switching of noncentrosymmetric compounds and $\mathrm{ZnO}$ structural properties. (a) The schematic diagram of ZnO hybrid switching structure, sandwiched by a top and a bottom electrode. (b) The classical resistance switching characteristic of the $\mathrm{ZnO}$ noncentrosymmetric materials. The resistance of noncentrosymmetric materials can be toggled between the low resistance state (LRS) and high resistance state (HRS) via electrical field stimulus. The SET (RESET) operation transform the HRS (LRS) of ZnO into LRS (HRS) when an applied voltage exceeds $\mathrm{V}_{\text {th,SET }}\left(\mathrm{V}_{\text {th,RESET }}\right)$. In the HRS regime, one can apply the electrical field across the sample and the polarization switching behavior can be studied. (c) Temperature dependence $\mathrm{x}$-ray diffraction (XRD) pattern of $\mathrm{ZnO}$ on Pt bottom electrode. The $\mathrm{ZnO}$ crystallinity improves with an increase of substrate temperature. Pronounced (002) peaks was observed for samples deposited at $400^{\circ} \mathrm{C}$ and $600^{\circ} \mathrm{C}$, respectively. A highly disordered amorphouslike structural of $\mathrm{ZnO}$ film was obtained for the samples prepared at the low deposition temperature of $30^{\circ} \mathrm{C}$.

switching) characteristic based on noncentrosymmetric compounds. The proposed structure is made of a noncentrosymmetric oxide film ( $\mathrm{ZnO}$ in our case), sandwiched by a top and a bottom electrode (Fig. 1a). The resistance state of the noncentrosymmetric materials can be toggled between the low resistance state (LRS) and high resistance state (HRS) via electrical field stimulus (Fig. 1b \& Fig. 2). Specifically, transformation from the LRS (HRS) to the HRS (LRS) of the noncentrosymmetric oxide film occurs via the SET (RESET) operation, when applied voltage exceeds $V_{\text {th,RESET }}\left(V_{\text {th,SET }}\right)$. Once in the HRS regime, one can apply the electrical field across the noncentrosymmetric oxide film and study the polarization switching behavior (Fig. 3). Here by 'switching', we refer to the reversal of the dipole moment in the crystal and subsequent retention of the polarization after the electrical field is removed. The ferroelectric-like domains in the noncentrosymmetric crystal can be polarized upward or downward in accordance to the direction of the electrical field.

Among the noncentrosymmetric compounds, zinc oxide $(\mathrm{ZnO})$ has been widely studied for its resistance switching ${ }^{15,16}$ and various switching mechanisms have been proposed ${ }^{1,3-7}$. Ferroelectric-like ${ }^{11-13}$ characteristics has been reported in doped-ZnO. Furthermore, $\mathrm{ZnO}$ is a material of technological importance due to its unique combination of piezoelectric, conductive, and optical properties ${ }^{17}$. $\mathrm{ZnO}$ is made up of alternative layers of $\mathrm{Zn}^{2+}$ and $\mathrm{O}^{2-}$ ions stacked in the [001]-direction, forming the $\mathrm{Zn}-\mathrm{O}$ bilayers (Figs. 4a-c). Each bilayer is stacked on top of another bilayer in the [001]-direction, in the stacking sequence ...AaBbAaBb...., forming a hexagonal closepacked structure. Within each bilayer, there exists a spontaneous polarization due to the net electric dipole moment pointing from $\mathrm{O}^{2-}$ to $\mathrm{Zn}^{2+}$. In this work, we demonstrate the non-volatile polarization / resistance switching effect in $\mathrm{ZnO}$ via a control of defect nanotechnology and contact engineering $(\mathrm{ZnO} / \mathrm{Pt})$. In addition to extensive experimental examinations, modelling and first-principles simulation were carried out to understand the physical origin of ferroelectric-like behaviour in $\mathrm{ZnO}$. Our computational studies indicate that oxygen vacancies $\left(\mathrm{V}_{\mathrm{O}}\right)$ play a key role in polarization switching. With the assistance of oxygen vacancies $\left(V_{O}\right)$, ferroelectric domain inversion initialises along $(1 \overline{1})$ grain boundary; the polarity of $\mathrm{Zn}$ and $\mathrm{O}$ atoms at adjoining position is inverted under an electrical field. More strikingly, these inverted domains can easily spread horizontally (in-plane) over a large area with a low energy barrier along an electrical field direction.

\section{Results}

To prove the concept of $\mathrm{ZnO}$ based hybrid memory, a series of $\mathrm{ZnO}$ films (nominal thickness $\sim 250 \mathrm{~nm}$ ) was deposited on various bottom electrodes $(\mathrm{Pt}, \mathrm{Au}, \mathrm{ITO}$, etc) by pulsed laser deposition technique (PLD) at a temperature between $30^{\circ} \mathrm{C}$ (room temperature) and $600^{\circ} \mathrm{C}$ under an oxygen partial pressure $\left(P_{o}\right)$ of $\sim 0.2 \mathrm{mTorr}$ (see Methods). All samples exhibit $n$-type semiconductivity with relatively high carrier concentration (see Supplementary Information, Table S1). Figure 1c shows a predominated $c$-axis (002) texture for $400^{\circ} \mathrm{C}$ and $600^{\circ} \mathrm{C}$ prepared $\mathrm{ZnO}$ films, corresponding to the $\mathrm{ZnO}$ wurtzite structure. It is noted that poor crystallinity was observed in films deposited at $30^{\circ} \mathrm{C}$. After experimenting with different deposition temperatures and various substrates, we realized that optimum $\mathrm{ZnO}$ microstructure can be achieved at deposition temperature between $200^{\circ} \mathrm{C}$ and $400^{\circ} \mathrm{C}$. The Pt substrate provides the better structural and resistance switching properties. The representative samples $-\mathrm{ZnO}$ on Pt electrode deposited at $400^{\circ} \mathrm{C}$ with a thickness of $250 \mathrm{~nm}$ were used for a detailed investigation. Figure 2 shows the local resistance switching of $\mathrm{ZnO} / \mathrm{Pt}$ deposited at $400^{\circ} \mathrm{C}$, as determined by conductive atomic force microscopy (C-AFM). The cyclic poling $(0 \mathrm{~V} \rightarrow 7 \mathrm{~V} \rightarrow-8 \mathrm{~V} \rightarrow 7 \mathrm{~V})$ was performed on the $\mathrm{ZnO}$ film over an area of $\left(5 \times 5 \mu \mathrm{m}^{2}\right)$ and the corresponding C-AFM images at specific points are displayed in Figs. $2 \mathrm{a}-\mathrm{g}$. The average current of each C-AFM image was determined by a commercial software (IGOR Pro 6) and its current density as a function of applied voltage $(J-V)$ is shown in Fig. 2h. The current starts to ramp up at $\sim 4 \mathrm{~V}$ and reaches its compliance limit $(10 \mathrm{nA})$ in the range of 5-6 V. Above $6 \mathrm{~V}$, the film possesses high current density with low resistance (LRS). In contrast, the film transforms to the high resistance state (HRS) with negligible current flow after application of $-6 \mathrm{~V}$ (RESET occurs). We noted that there is a negligible current flow across the film even when a higher voltage of $-8 \mathrm{~V}$ is applied after RESET 

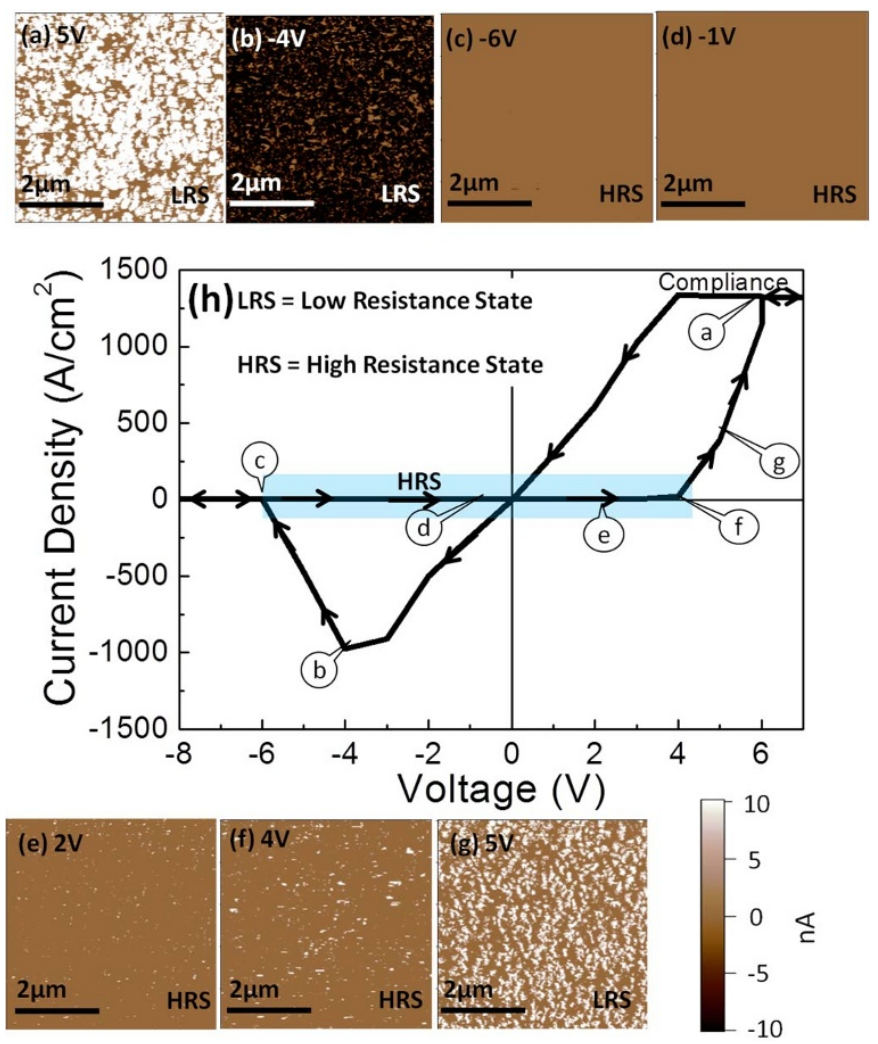

Figure $2 \mid$ The resistance switching characteristic of $\mathrm{ZnO}$ by conductive atomic force microscope (C-AFM) analysis. ( $\mathrm{a}-\mathrm{g}$ ) Using a conducting $\mathrm{Pt}$ tip, a cyclic poling $(0 \mathrm{~V} \rightarrow 7 \mathrm{~V} \rightarrow-8 \mathrm{~V} \rightarrow 7 \mathrm{~V})$ was performed on $\mathrm{ZnO}$ films $\left(5 \times 5 \mu \mathrm{m}^{2}\right)$ and C-AFM images were captured. The average current of each C-AFM image was analysed by commercial software (IGOR Pro 6) and its current density is plotted in $\mathbf{h}$ as function of applied voltage. Apparently, two distinct regimes (LRS and HRS) were observed. Above $6 \mathrm{~V}$, the film possesses high current density with low resistance state (LRS). In contrast, the film transforms to high resistance state (HRS) with negligible current flow after application of $-6 \mathrm{~V}$. The film retains its HRS state between

$-6 \mathrm{~V}$ to $+4 \mathrm{~V}$ and this regime is particular important for its polarization switching study. It is noted that voltage is applied to the Pt substrate with tip grounding based on our C-AFM configuration.

operation. This resistance switching phenomenon is repeatable in other regions of the samples as well as other samples (x3). In fact, the observed bipolar resistance switching is consistent with previous experimental results ${ }^{3,18}$. In the $\mathrm{ZnO}$ films being studied, the HRS regime ranged from $-8 \mathrm{~V}$ to $+4 \mathrm{~V}$ after the RESET operation at $-6 \mathrm{~V}$ (Fig. 2h). To confirm the Pt tip working condition, the Pt tip condition was checked after each SET $\rightarrow$ RESET $\rightarrow$ SET cyclic scanning. The similar $1 \mathrm{~V}$ C-AFM images were obtained after each SET operation as compared to its initial $1 \mathrm{~V}$ C-AFM image (reference), confirming the Pt tip was in good working mode before and after CAFM measurement (see Supplementary Information, Fig. S1).

To study the involvement of the polarization switching, piezoresponse force microscope (PFM) measurements were carried out (Fig. 3). It is noted that the electrical field of PFM machine was applied to the tip with respective to substrate grounding, which is opposite to the C-AFM measurement described previously. Conversely, the applied voltage of the C-AFM is applied on substrate with Pt tip grounding. Thus, a positive bias in PFM corresponds to a negative bias in C-AFM and vice versa. To evaluate the effectiveness of HRS regime under PFM setup, we have examined the polarization switching of the $\mathrm{ZnO}$ film deposited on Pt electrode at $400^{\circ} \mathrm{C}$ by PFM system (Fig. 3). Our PFM results have shown that an applied voltage range between $+6 \mathrm{~V}$ and $-3 \mathrm{~V}$ is sufficient for the polarization switching investigation with high reproducibility. Therefore, a poling voltage range of $+6 \mathrm{~V}$ and $-3 \mathrm{~V}$ was used in the PFM experiments and most of PFM discussions below are based on these poling voltages.

Prior to PFM analysis, the $\mathrm{ZnO}$ samples were transformed into the HRS regime using the method described above (Fig. 2h). Then using the conductive $\mathrm{Pt}$ tip, the high resistive $\mathrm{ZnO}$ film was poled by $-3 \mathrm{~V}$ (Fig. 3a) and $+6 \mathrm{~V}$ (Fig. 3c), respectively over an area of $5.5 \times 5 \mu \mathrm{m}^{2}$. A remarkable contrast between the unpoled and poled region was observed. The poling results reveal the flipping of domains to upward (downward) direction under the application of a negative (positive) voltage, indicating upward (downward) polarization has been written in the $\mathrm{ZnO}$ film. It is also noted that most of the domains were pointing upward at its initial (unpoled) state (see Supplementary Information, Fig. S2b). The corresponding PFM amplitude image (see Supplementary Information, Fig. S2f) depicts volume expansion after negative and positive poling, as supported by amplitude hysteresis loops (see Supplementary Information, Fig. S2g). It is noted that electrical poling has insignificant effect on the surface topography (see Supplementary Information, Fig. S2d), reflecting the film surface is not damaged after poling. More importantly, the polarization in these domains can be manipulated by an electrical field. A remarkable alternative stripes with clear contrast was appeared after a subsequent $+6 \mathrm{~V}$ poling on top of existing $-3 \mathrm{~V}$ poling pattern (Fig. 3b). The prominent PFM phase contrast difference $\left(\sim 180^{\circ}\right)$ between negatively and positively poled regimes is an indication to $\mathrm{ZnO}$ pseudoferroelectricity at room temperature. It is worthwhile to highlight that the PFM poling effect is sensitive to its poling conditions (see Method).

To get further insight into the polarization sustainability (retention time), the time dependence PFM imaging was performed. The $\mathrm{ZnO}$ film was first poled by $+6 \mathrm{~V}$, which resulted in a remarkable dark contrast (downward polarization) as shown in Fig. 3c. Then PFM images were taken after 2 hours (see Supplementary Information, Fig. S3c) and 25 hours (Fig. 3d) of poling respectively, both images elucidate the prominent contrast difference between poled and unpoled regimes. Taking PFM images obtained after 2 hours of poling as a reference, statistical results show that more than $90 \%$ of polarised domains retained their polarization after 25 hours (see Supplementary Information, Fig. S3e). This longlasting PFM phase contrast in the high resistive $\mathrm{ZnO}$ film excludes the charging effect as a possible origin ${ }^{19}$. To further investigate the origin of polarization, the sample was re-scanned after the removal of surface static charge by static-removal device. An insignificant change in PFM phase contrast was found after the surface static charge removal process (see Supplementary Information, Fig. S4). Furthermore, the ground tip scanning experiment was carried out. The polarization of $\mathrm{ZnO}$ has been retained after the ground tip scanning across the poled regimes. These observations essentially rule out the role of charging effect in the polarization switching study.

To further probe the polarization switching phenomenon in $\mathrm{ZnO}$, we performed Piezoresponse Force Spectroscopy (PFS) measurement $^{20}$ (see Methods). Figure $3 \mathrm{e}$ shows the voltage dependence of phase hysteresis loop with phase difference of $180^{\circ}$, which is coherent with PFM phase images. A "butterfly shape" piezoresponse amplitude signal was clearly seen in Fig. 3f. It is noted that the amplitude becoming unstable with reduction in its magnitude beyond $6 \mathrm{~V}$ and $-4 \mathrm{~V}$. Such a phenomenon may be associated to the high current flow in the film that is detrimental to the piezoresponse of $\mathrm{ZnO}$. These PFS hysteresis loops were highly reproducible, as evidenced by our repetitive switching analysis (see Supplementary Information, Fig. S2g and 2h). The concomitant observation of the PFS loops with $180^{\circ}$ phase change and "butterfly shape" piezoresponse signals in the high resistive $\mathrm{ZnO}$ films attest their polarization switching and pseudoferroelectricity character at room temperature.

Furthermore, we have studied the polarization switching of the $\mathrm{ZnO}$ samples deposited at various temperatures. The thickness of 

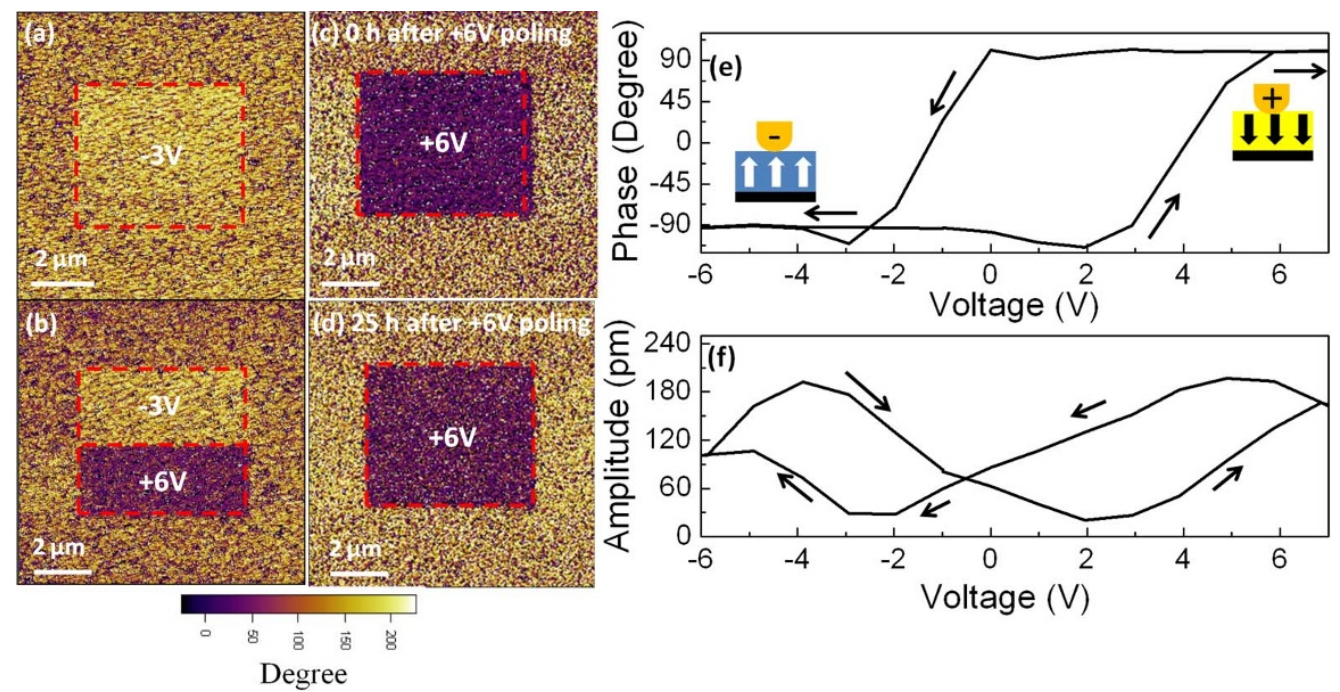

Figure $3 \mid$ Direct evidence of polarization switching and pseudoferroelectric properties of $\mathrm{ZnO}$ on Pt electrode. Using a conducting Pt tip, the polarization switching of $\mathrm{ZnO}$ is studied by PFM technique. (a) The PFM phase images of $\mathrm{ZnO}\left(10 \times 10 \mu \mathrm{m}^{2}\right)$ after application $-3 \mathrm{~V}$ over region of $5.5 \times 5 \mu \mathrm{m}^{2}$ (red dotted regime). (b) A remarkable alternating stripes $\left(5.5 \times 2.5 \mu \mathrm{m}^{2}\right)$ is seen after subsequent $+6 \mathrm{~V}$ poling on top of the existing $-3 \mathrm{~V}$ poling pattern, elucidating the $180^{\circ}$ phase change and it is supported by (e) PFM phase hysteresis loop. The corresponding piezoresponse signal of ZnO shown in $\mathbf{f}$ depicts the "butterfly shape". It is noted that the amplitude loop getting unstable with reduction in magnitude above $6 \mathrm{~V}$ and $-4 \mathrm{~V}$. (c,d) The polarization retention time study, showing majority of polarization are retained after 25 hours. It is noted that voltage is applied to tip with substrate grounding based on our PFM configuration.

$\mathrm{ZnO}$ was kept constant at $250 \mathrm{~nm}$. It was found that $\mathrm{ZnO}$ deposited at $400^{\circ} \mathrm{C}$ on Pt electrode exhibited a uniform and stable polarization switching (see Supplementary Information, Fig. S5b). In stark contrast, the $\mathrm{ZnO}$ film deposited at room temperature $\left(30^{\circ} \mathrm{C}\right)$ shows a very poor electric poling response (see Supplementary Information, Fig. S5a). Similarly, the sample deposited at the higher temperature of $600^{\circ} \mathrm{C}$ does not show a uniform polarization switching (see Supplementary Information, Fig. S5c). The corresponding enlarged view shows that most dark areas (polarized areas) are concentrated along grain boundaries (see Supplementary Information, insert of Fig. S5c). Figure S6a (see Supplementary Information) shows the statistical distribution of polarized area (see Method) of $\mathrm{ZnO}$ film deposited at various temperature, as poled by $+6 \mathrm{~V}$ over region of $5 \mu^{2}$. Three district polarization regimes were identified. Apparently, $\mathrm{ZnO}$ films deposited at 200,300 and $400^{\circ} \mathrm{C}$ (Regime II) exhibit the strongest polarization with $>90 \%$ polarizable domains (see Supplementary Information, Fig. S6a). It is noted that these films were well-crystallized with a (002) texture (Fig. 1c) and grain sizes around $50 \mathrm{~nm}$, as seen by transmission electron microscopy (TEM) analysis. In contrast, weak polarization was detected in the samples deposited at lower temperature $\left(30^{\circ} \mathrm{C}\right.$ to $100^{\circ} \mathrm{C}$; Regime I) and higher temperature $\left(500^{\circ} \mathrm{C}\right.$ to $600^{\circ} \mathrm{C}$; Regime III).

To have a clearer picture, we examined the resistivity switching and micro-structural properties of $\mathrm{ZnO}$ deposited at $30^{\circ} \mathrm{C}$ and $600^{\circ} \mathrm{C}$, respectively. The $\mathrm{ZnO}$ samples deposited at $30^{\circ} \mathrm{C}$ do not exhibit a high resistance state (HRS) (see Supplementary Information, Fig. S7a) due to high current leakage at high voltage regime. The nature of low resistance and high electron concentration $\left(8 \times 10^{19} \mathrm{~cm}^{-3}\right)$ of $30^{\circ} \mathrm{C}$ prepared $\mathrm{ZnO}$ (see Supplementary Information, Table S1) suppressed the effectiveness of an electric field applies onto the samples, leading to weak pseudoferroelectricity. The microstructure analysis of $30^{\circ} \mathrm{C}$ prepared film by TEM reveals an amorphous with highly disordered structure (see Supplementary Information, Fig. S6b), which is consistent with our XRD data (Fig. 1c).

On the other hand, the $\mathrm{ZnO}$ deposited at $600^{\circ} \mathrm{C}$ possesses a reasonably good high resistance state (HRS) after the RESET operation of $-5 \mathrm{~V}$ in negative voltage regime (see Supplementary Information,
Fig. S7b). However, the HRS characteristic failed to expend into the positive voltage regime. A relatively large leakage current was observed when applied voltage exceeded $+1 \mathrm{~V}$. We noted that these samples exhibited superior structural properties with larger grain size $(150 \mathrm{~nm}$ to $200 \mathrm{~nm})$ and sharp interface between neighboring grains (see Supplementary Information, Fig. S6c). To further examine its microstructure, we performed the AFM and C-AFM imaging of $600^{\circ} \mathrm{C}$ prepared $\mathrm{ZnO}$ films. By proper reconstructing the AFM and C-AFM images (see Supplementary Information, Fig. S7c \& d), we found that the film was rather leaky and most of current leaking areas (white areas) were located near the grain boundaries. Such leaky phenomenon lead to incomplete and weak polarization for the $600^{\circ} \mathrm{C}$ prepared $\mathrm{ZnO}$ samples. This is because parts of grain boundaries do not response to the applied electrical field due to the electron screening effect. These results seems to imply that large grain size is not favorable for polarization switching in $\mathrm{ZnO}$ whereas a nanocrystalline structure $\left(\sim 50 \mathrm{~nm}\right.$ for $400^{\circ} \mathrm{C}$ prepared samples) is desirable for pseudoferroelectricity in $\mathrm{ZnO}$.

Moreover, we have studied the thickness dependence pseudoferroelectricity of $\mathrm{ZnO}$, down to $15 \mathrm{~nm}$. All the films were deposited at $400^{\circ} \mathrm{C}$. The thinner films were found to exhibit higher percentage of switchable domains (see Supplementary Information, Fig. S11a). In particular, the $\mathrm{ZnO}$ films with $15 \mathrm{~nm}$ and $30 \mathrm{~nm}$ thick have nearly $100 \%$ of the polarizable domains. The polarization of thicker films $(>500 \mathrm{~nm})$ with larger grain size $(>100 \mathrm{~nm})$ displayed weakened pseudoferroelectricity. These results further confirm the importance of nanocrystalline structure in the realization of $\mathrm{ZnO}$ pseudoferroelectricity.

Since polarization switching is impossible in perfect $\mathrm{ZnO}$ crystal (see Supplementary Information, Section B and Fig. S9), defects are expected to play a critical role in polarization switching and pseudoferroelectricity. Native defects such as oxygen vacancies $\left(\mathrm{V}_{\mathrm{O}}\right)$ are commonly observed in $n$-type $\mathrm{ZnO}$. The presence of $\mathrm{V}_{\mathrm{O}}$ in our films is further supported by the appearance of broad $E_{1}(\mathrm{LO})$ peak $\left(\sim 587 \mathrm{~cm}^{-1}\right)$ in the Raman spectrum and detection of green emission (centred at $\sim 500 \mathrm{~nm}$ ) in photoluminescence analysis (see Supplementary Information, Fig. S8). 


\section{Basic $\mathrm{ZnO}$ units}

(a)

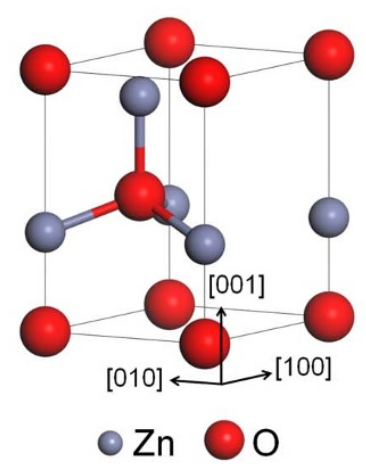

(b)

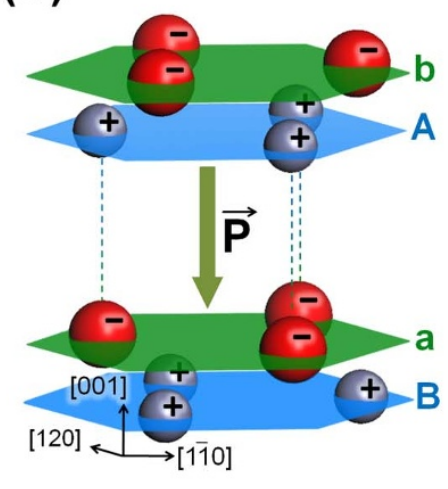

Twin Grain Boundary

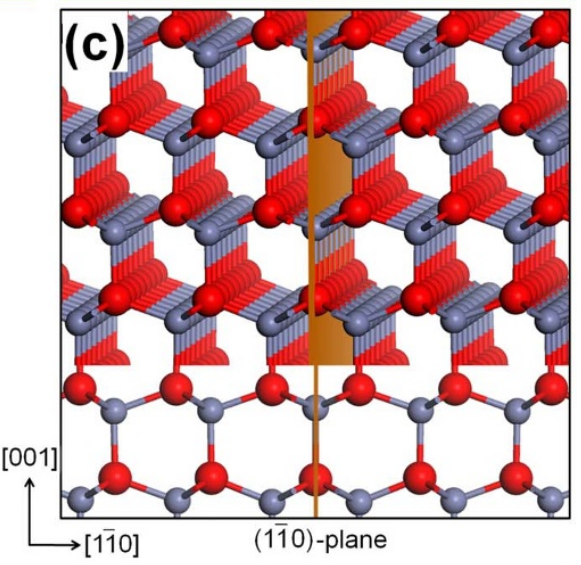

Inversion Domain Wall (IDW*)

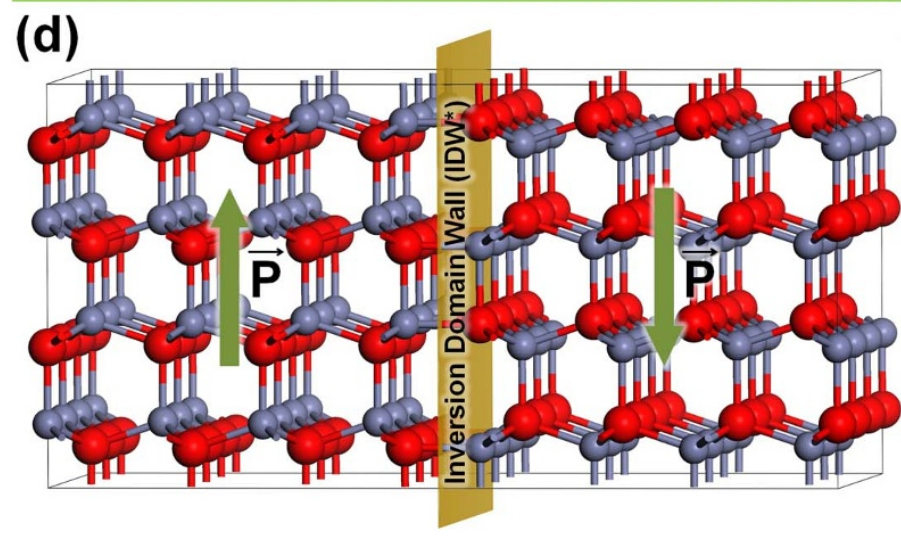

(e)

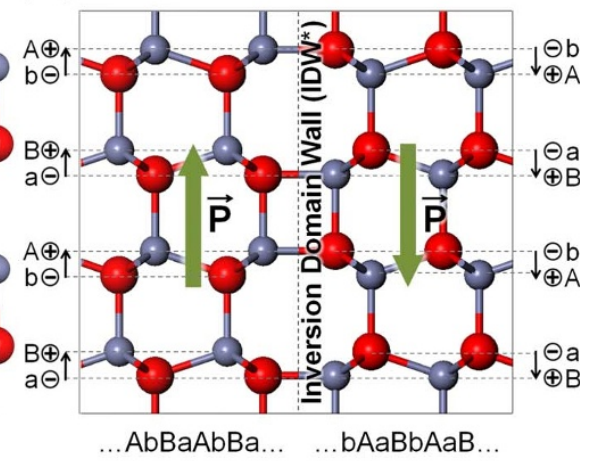

Figure $4 \mid$ The atomistic model of wurtzite $\mathrm{ZnO}$, a twin grain boundary and an inversion domain wall (IDW*). (a) $\mathrm{ZnO}$ unit cell, including the tetrahedral-coordination between $\mathrm{Zn}$ and its neighboring $\mathrm{O}$. (b) $\mathrm{ZnO}$ has a noncentrosymmetric crystal structure that is made up of alternate layers of positive and negative ions, leading to spontaneous polarization $\vec{P}$. The pseudoferroelectric switching involves the reversal of these dipole moments. (c) A low-energy twin ( $1 \overline{1} 0)$ boundary that was used to model the grain boundary. (d) A 3D representation of the domain wall in the (1 $\overline{1} 0)$ plane, separating two oppositely-polarised $\mathrm{ZnO}$ domains. (e) The corresponding $2 \mathrm{D}$ representation of $\mathbf{d}$.

To understand the underlying mechanism of polarization switching in $\mathrm{ZnO}$, we carried out first-principles simulation. It is recalled that our experimental results have shown the importance of the nanocrystalline structure and $\mathrm{V}_{\mathrm{O}}$ towards $\mathrm{ZnO}$ pseudoferroelectricity. In addition, the results suggest that polarization switching is initiated at grain boundaries. Taking all experimental observations into consideration, we performed extensive modeling and first-principles calculations based on the Density Functional Theory (DFT) as implemented in the Vienna ab-initio simulation package (VASP) (See Methods). Accordance to our experimental observations that $\mathrm{ZnO}$ possesses a predominant (002) texture and most of the domain walls are perpendicular to the film, thus we modeled the domain wall with the (110) plane. It is perpendicular to the (002) plane and is commonly present in $\mathrm{ZnO}$ sys$t^{21,22}$. The (110) is also known as the m-plane for wurtzite $\mathrm{ZnO}$ and is symmetrically equivalent to and representative of a family of domain boundaries, including $(\overline{1} 10),(010),(100),(0 \overline{1} 0)$ and $(\overline{1} 00)^{22,23}$.

Specifically, a twin boundary (Fig. 4c) was used to model the grain boundary structure as it was found to have a low formation energy of $0.06 \mathrm{eV} / \AA^{2}$. The formation energy of an oxygen vacancy $\left(\mathrm{V}_{\mathrm{O}}\right)$ at the grain boundary was first evaluated and compared to that in bulk $\mathrm{ZnO}$. The results signify a thermodynamic preference of oxygen vacancy to form at the grain boundary because its formation energy at the grain boundary is always lower than that in bulk by about $0.44 \mathrm{eV}$ (see Supplementary Information, Fig. S10). Subsequently, we carried out modeling and calculations to investigate the pseudoferroelectricity in $\mathrm{ZnO}$ in three key steps. First, a three-dimensional (3D) $\mathrm{ZnO}$ inversion domain model was developed and evaluated. Second, the switching mechanism of inverted domains along the (110) boundary was studied. Finally, the propagation of $\mathrm{ZnO}$ pseudoferroelectric domains over large areas via domain wall movement was evaluated.

$\mathrm{ZnO}$ unit cell possesses a spontaneous electric dipole moment and its orientation is along the [001]-direction as shown in Fig. $4 \mathrm{~b}$. Certainly, such an electric dipole moment is not the same as that in typical ferroelectric materials, such as $\mathrm{BaTiO}_{3}$, in which polarization switching is realized by the movement of the smaller Ti cations about off-center position ${ }^{23}$. Therefore, the ferroelectric-like behaviour found in $\mathrm{ZnO}$ is termed as "pseudoferroelectricity". Our experimental results showed that the $\mathrm{ZnO}$ film is highly textured in the predominant [001]-direction (Fig. 1c) and a scanning PFM tip can polarize $\mathrm{ZnO}$ upwards in the [001]-direction or downwards in the [001] -direction. $\mathrm{ZnO}$ can thus be said to adopt either of the two orientational states (Fig. 4d): upwards or downwards. In between two oppositely polarized domains, an inversion domain wall (IDW*) exists and its structure has been previously proposed ${ }^{21,24}$. Our calculation confirms that an inversion domain wall as shown in Fig. $4 \mathrm{~d}$ has a formation energy of $0.01 \mathrm{eV} / \AA^{2}$. Figures $4 \mathrm{~d}$ and $4 \mathrm{e}$ mark out the $\mathrm{ZnO}$ dipole moments polarized upwards in the [001]-direction and downwards in the $[00 \overline{1}]$-direction and separated by an inversion domain wall $(\mathrm{IDW} *)$. 


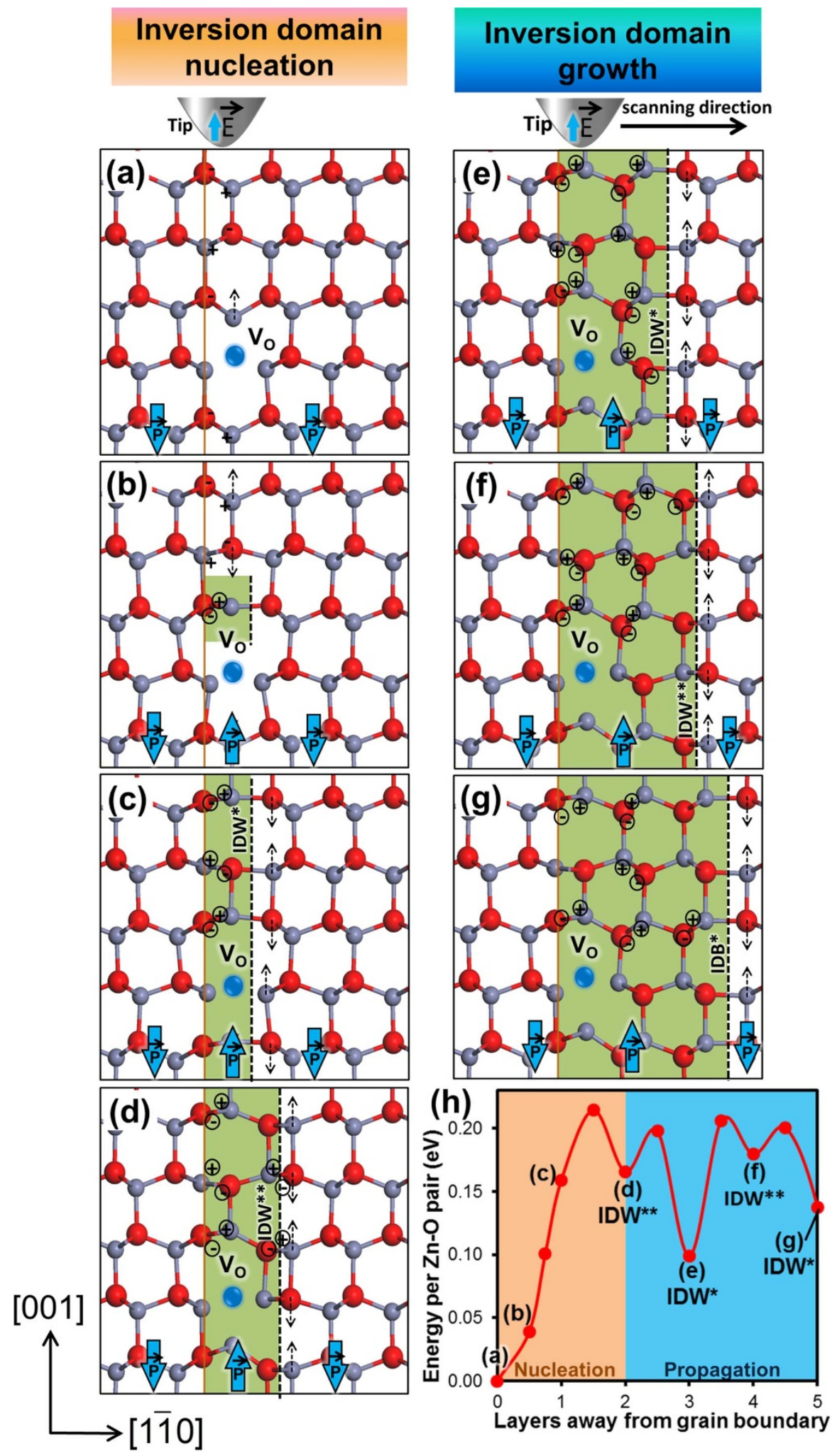

Figure $5 \mid$ Outline of pseudoferroelectric domain nucleation, propagation and growth. (a-d) Nucleation of a pseudoferroelectric domain at a ZnO (1 $\overline{1} 0)$ boundary. (a) Due to the presence of the $\mathrm{V}_{\mathrm{O}}$, the $\mathrm{Zn}$ atom above lost its bonding to the missing oxygen. (b) Under an electric field, the positively charged $\mathrm{Zn}$ ion moves up in the vertical direction (c), the polarity of $\mathrm{Zn}$ and $\mathrm{O}$ atoms at the adjoining position is then inverted and the inversion spreads vertically along the boundary rapidly, leaving a trail of inversion ferroelectric domain in its wake and forming an inversion domain wall (IDW*) along the grain boundary. (d) In the presence of an external electric field, $\mathrm{Zn}^{2+}$ and $\mathrm{O}^{2-}$ at the IDW* experience an electric force and are displaced in opposite directions by $0.5 \AA$, relieving the strain in its four-membered ring forming an IDW**. (e-g) The propagation of pseudoferroelectric domain wall from left to right in the presence of an external electric field. (e) An electric force on $\mathrm{Zn}^{2+}$ and $\mathrm{O}^{2-}$ at the $\mathrm{IDW}^{* *}$ once again displaces $\mathrm{Zn}^{2+}$ and $\mathrm{O}^{2-}$ in opposite directions by about $0.5 \AA$, regenerating an IDW $^{*}$. (e-g) Propagation of the inversion domains wall in the sequence $\cdots$ IDW $^{*} \rightarrow$ IDW $^{* *} \rightarrow$ IDW $^{*} \cdots(\mathrm{h})$ Energy profile of the nucleation and propagation of the pseudoferroelectric domain. The dotted arrows represent the displacement direction of $\mathrm{Zn}$ and $\mathrm{O}$ atoms under application of electrical field. 
Similar to classic ferroelectric materials, one can expect the polarization switching in $\mathrm{ZnO}$ to be initiated by the formation of the very first inverse domain wall followed by the spreading of the inversion domain to complete the depolarization via domain wall propagation. Thus, we have performed a careful study on the formation of an inverse pseudoferroelectric domain. It is found that inverse domain cannot be formed in perfect $\mathrm{ZnO}$ crystal because the energy barrier of ion diffusion is too high (over $5 \mathrm{eV}$ per $\mathrm{Zn}-\mathrm{O}$ pair). The details $\mathrm{ZnO}$ pseudoferroelectric evaluation are available in Section B of Supplementary Information, Fig. S9. Therefore, we considered a more realistic scenario - the polarization in $\mathrm{ZnO}$ is assisted by $\mathrm{V}_{\mathrm{O}}$ defects as $\mathrm{V}_{\mathrm{O}}$ is abundant in the $\mathrm{ZnO}$ samples, as evidenced by Raman, PL and Hall-effect measurements.

Since our first-principles calculations indicated that $\mathrm{V}_{\mathrm{O}}$ is more likely to form at grain boundaries (see Supplementary Information, Fig. S10), we developed a model that involves the nucleation of an inversion domain along the grain boundary assisted by $\mathrm{V}_{\mathrm{O}}$. Figures $5 a-d$ show a schematic representation of the nucleation process of a pseudoferroelectric domain which is induced by $V_{O}$ at the (110) boundary. Due to the presence of the $\mathrm{V}_{\mathrm{O}}$, the $\mathrm{Zn}$ atom above lost its bonding to the missing oxygen (Fig. 5a). Under the applied electric field, the positively charged $\mathrm{Zn}$ ion moves up in the vertical direction (Fig. 5b). The polarity of $\mathrm{Zn}$ and $\mathrm{O}$ atoms at adjoining position is then inverted and the inversion spreads vertically along the boundary rapidly, leaving a trail of inverted pseudoferroelectric domain in its wake and forming an inversion domain wall (IDW*) along the grain boundary (Fig. $5 \mathrm{c}$ ).

Next, we will evaluate the propagation of inversion domains wall over large area. Once an inversion domain is formed, it can propagate easily and laterally in the direction of the scanning tip under the presence of electrical field. Due to the external electric field applied in the [001]-direction, $\mathrm{Zn}^{2+}$ and $\mathrm{O}^{2-}$ at the inversion domain wall $\left(\mathrm{IDW}^{*}\right.$ ) experience an electric force (Fig. 5c) and are displaced in opposite directions by about $0.5 \AA$ in response to the applied field (Fig. 5d). This relieves the strain in its four-membered ring while forming another four-membered ring at a new boundary structure, denoted here as IDW** (Fig. 5d). IDW** is structurally similar to $\mathrm{IDW}^{*}$, except for the minor displacement of $\mathrm{Zn}-\mathrm{O}$ pairs as described above. Upon formation of this new intermediate boundary structure $\mathrm{IDW}^{* *}$, the domain wall has propagated in that same direction $\left(\mathrm{IDW}^{*} \rightarrow \mathrm{IDW}^{* *}\right.$ ) (Fig. 5c $\rightarrow$ Fig. 5d). In the energy profile (Fig. 5h), we see that this configuration corresponds to a local energy minimum. The nucleation of inversion domain under an applied electrical field has a low energy barrier of $\sim 0.2 \mathrm{eV}$ per $\mathrm{Zn}-\mathrm{O}$ pair.

Sustained application of an electric field in the scanning direction then causes the inversion domain wall to propagate. $\mathrm{Zn}^{2+}$ and $\mathrm{O}^{2-}$ at the IDW ${ }^{* *}$ experience an electric force (Fig. $5 \mathrm{~d}$ ), once again displacing $\mathrm{Zn}^{2+}$ and $\mathrm{O}^{2-}$ in opposite directions by about $0.5 \AA$ (Fig. 5e). This similarly relieves the strain in the 4 -membered ring at the $\mathrm{IDW}^{* *}$ and re-forms an $\mathrm{IDW}^{*}$ in the propagation direction $\left(\right.$ IDW $^{* *} \rightarrow$ IDW $^{*}$ ) (Fig. $5 \mathrm{~d} \rightarrow$ Fig. 5e), driving the propagation further. Subsequently, the inversion domains wall propagates continuously in the sequence $\cdots$ IDW $^{*} \rightarrow$ IDW $^{* *} \rightarrow$ IDW $^{*} \cdots$ (Fig. 5e $\rightarrow$ Fig. 5g), which is facilitated by the cyclic relief of strain in fourmembered rings. In response to the electric field, the pseudoferroelectric domain is polarized in the direction parallel to the applied field and grows in size. As the PFM tip scans across the (001)-plane, the domain wall propagates continuously, switching electric dipoles along the way, until it meets another wall. Two neighboring pseudoferroelectric domains then coalesce to form one larger domain. As shown in Fig. 5h, the largest propagation barrier is only $\sim 0.1 \mathrm{eV}$ per $\mathrm{Zn}-\mathrm{O}$ pair.

\section{Discussion}

As discussed above, our experimental results indicate that grain size plays an important role in $\mathrm{ZnO}$ pseudoferroelectricity. Smaller grain size enables an effective polarization switching, while larger grain size is accompanied by a partial polarization switching with a significant leakage current (see Supplementary Information, Fig. S7b). Analogy to the concept of single domain particle size in ferromagnetic materials ${ }^{24}$, we could obtain the value around $25 \mathrm{~nm}$ for "single domain pseudoferroelectric $\mathrm{ZnO}$ particle" based on the minimization of domain wall energy and dielectric field energy. This theoretical grain size is closer to the experimental grain size $(\sim 50 \mathrm{~nm})$ of $\mathrm{ZnO}$ film that prepared at a temperature range of $200-400^{\circ} \mathrm{C}$. The formation ability of domain wall with grain size is also estimated based on $1^{\text {st }}$ principle calculations. It is found that domain wall could occur spontaneously at room temperature in the grain with size larger than $25 \mathrm{~nm}$. The density-of-state (DOS) of $\mathrm{ZnO}$ with a domain wall structures suggest that domain walls have a high conductivity (metal-like transport properties), as the Fermi level lies in the conduction band. That explain why large grain size is not favourable for polarization switching due to spontaneously formation of conductive domain wall. It is in agreement with the experiment observations that current leakage is located near the grain boundary (see Supplementary Information, Fig. S7d). In fact, our result is consistent with the classic ferroelectric materials that possess higher conductivity in the domain wall ${ }^{25}$. The domain wall conductivity in a ferroelectric materials has been shown via numerical calculation previously ${ }^{26}$. Such conductive domains wall leads to weakened pseudoferroelectricity in the $\mathrm{ZnO}$ sample with a larger grain size.

In conclusion, we have demonstrated room temperature nonvolatile polarization and resistance switching in $\mathrm{ZnO}$ via a deterministic control of the oxygen vacancies, carrier concentration, nano-grain structure, grain boundaries and contact engineering. The pronounced resistance switching behaviors (low resistance state (LRS) $\leftrightarrow$ high resistance state (HRS)) was observed in $\mathrm{ZnO}$ films prepared at $400^{\circ} \mathrm{C}$ with its average grains size of $50 \mathrm{~nm}$. In the HRS regime, $\mathrm{ZnO}$ domains can be polarized with $180^{\circ}$ phase switching along the [001] axis by an electrical field. The polarization was longlasting (more than 25 hours) and ferroelectric domains could be written and erased repetitively. An oxygen vacancy $\left(\mathrm{V}_{\mathrm{O}}\right)$ mediated pseudoferroelectricity model is proposed to explain satisfactorily the nucleation and propagation of inversed domain. Based on this model, polarization switching initiates at the inversion domain boundaries $\left(\mathrm{IDW}^{*}\right.$ ) in the presence of $\mathrm{V}_{\mathrm{O}}$, such that the $\mathrm{Zn}$-O polarity is inverted along the $(1 \overline{1} 0)$ boundary under an applied electric field. The nucleation of inversion domain under an applied electrical field has a low energy barrier of $\sim 0.2 \mathrm{eV}$ per $\mathrm{Zn}-\mathrm{O}$ pair. With a mutual complement of the spontaneous domains nucleation and propagation, $\mathrm{Zn}^{2+}$ and $\mathrm{O}^{2-}$ ionic pair with an energy barrier of $0.1 \mathrm{eV}$ will migrate and switch along the direction of the applied electric field, leading to the growth of inverted domains over a large area. This accomplishment offers exciting opportunities for the fabrication of $\mathrm{ZnO}$ multiple bits information storage that is compatible with today's silicon technology.

\section{Methods}

Films preparation and characterizations. ZnO powders (99.99\%, Sigma-Aldrich) are used in the present study to make an undoped $\mathrm{ZnO}$ pallet, then it was sintered at $1000^{\circ} \mathrm{C}$ for 12 hours to obtain $\mathrm{ZnO}$ target. We have carried out an extensive parameters optimization to obtain superior crystalline $\mathrm{ZnO}$ films with a prominent $c$ axis (002) texture (Fig. 1c). With these optimum conditions, a series of $\mathrm{ZnO}$ films with a nominal thickness of $250 \mathrm{~nm}$ was prepared on Pt bottom electrode by pulsed laser deposition (PLD) using a $\mathrm{KrF}$ excimer laser operating at $248 \mathrm{~nm}$ and a fluence of $1.8 \mathrm{~J} \mathrm{~cm}^{-2}$, ranging from room temperature $\left(30^{\circ} \mathrm{C}\right)$ to $600^{\circ} \mathrm{C}$ under oxygen partial pressure of 0.2 mTorr. Our study shows that $\mathrm{ZnO}$ crystallinity and structural properties are sensitive to deposition temperature. The low temperature $\left(<200^{\circ} \mathrm{C}\right)$ deposited $\mathrm{ZnO}$ films possessed the amorphous-like structure. To have an in-depth understanding of $\mathrm{ZnO}$ ferroelectricity, we carried out the thickness dependence study. A series of $\mathrm{ZnO}$ film with various thicknesses was deposited on the platinum $(\mathrm{Pt})$ bottom electrode $\left(\mathrm{ZnO}\right.$ deposited on $\mathrm{Pt} / \mathrm{Ti} / \mathrm{Si} / \mathrm{SiO}_{2}$ substrate) under oxygen partial pressure of $0.2 \mathrm{mT}$ orr at $400^{\circ} \mathrm{C}$, ranging from $15 \mathrm{~nm}$ to $600 \mathrm{~nm}$. The structural properties improved with an increase film thickness, but degradation in pseudoferroelectricity. To obtain uniform oxygen distribution across the films, the samples were kept at identical deposition temperature and oxygen partial pressure for 
$\sim 30$ mins prior to cooling down. Then the samples were slowly cooled down to room temperature under oxygen ambient (partial pressure $\sim 0.2 \mathrm{mtorr}$ ). It is worthwhile to highlight that all the samples were sealed by parafilm immediately after the deposition and kept in a dry box, in order to avoid oxidation.

The structural properties of the samples were studied by X-ray diffraction (XRD; Briker AXS D8 Advance), TEM (Joel, JEM 2010F) and Raman spectroscopy (LabRam HR 800). The green emission was analyzed by the integrated photoluminescence system (JY-Horiba, FluoroLog-3 spectrofluorometer) under excitation of $325 \mathrm{~nm}$ He-Cd laser source $(30 \mathrm{~mW})$.

Resistance switching analysis. The local resistance switching behaviors were investigated by conductive atomic force microscope (Asylum Research), using a commercially available Pt-coated tips. Conductive Pt tips (AC240TM Olympus, Japan) with $15 \mathrm{~nm}$ tip radius, $2 \mathrm{~N} / \mathrm{m}$ stiffness and its resonance frequency of $70 \mathrm{kHz}$ was used. The commercial software (IGOR PRO 6.12A) together with the Asylum Research code (version 090909-1214) was used to get the average current distribution of C-AFM image. An average current density as function of voltage is plotted.

Pseudoferroelectric properties. A commercial vertical Piezoresponse force microscopy (VPFM, MFP-3D, Asylum Research, USA) is used to characterize the ferroelectric and local switching behaviours of $\mathrm{ZnO}$ film. Conductive tips (AC240TM, Electric-Lever, Olympus, Japan) with $15 \mathrm{~nm}$ tip radius, $2 \mathrm{~N} / \mathrm{m}$ stiffness and its resonance frequency of $70 \mathrm{kHz}$ was used. It is worthwhile to highlight that the poling process is sensitive to scan speed, poling voltage, and the contact between the tip and $\mathrm{ZnO}$ film. Thus, extensive optimizations have been performed to get the reliable and best PFM images. For the dc poling, a line scanning method with a scanning speed of $12.5 \mu \mathrm{m} / \mathrm{s}$, response drive amplitude of $1 \mathrm{~V}$, set point of $0.6 \mathrm{~V}$ and frequency of $1 \mathrm{~Hz}$ frequency was employed. Furthermore, poling effect is depended on the tip Pt coating quality. The Pt tip with a resonant frequency $\sim 70 \mathrm{kHz}$ and work function difference of $\sim 500 \mathrm{mV}$ (between $\mathrm{ZnO}$ and Pt tips, as determined by Kelvin Probe Force Microscopy (KPFM) technique) always offers good poling results. It is also noted that samples oxidation may affect the PFM analysis, thus all the samples were sealed and kept in the dry box immediately after the deposition.

In Piezoresponse Force Spectroscopy (PFS) ${ }^{20}$ investigation, a sine-like triangular waveform that carried a sequence of square waves with its frequency of $400 \mathrm{mHz}$ was applied to the tip. The remnant bias-induced polarization is acquired at every pulse between the adjacent voltage steps when the bias is stepped back to zero.

The commercial software (IGOR PRO 6.12A) together with the Asylum Research code (version 090909-1214) was used to get the statistical distribution of switched domains. This operating system captures the PFM scanned information (such as piezoresponse, polarization, etc.) of each pixel. This information can be further used for the statistic distribution calculation.

First-principles calculation and modeling. To model the ferroelectric phase transition, we carry out first-principles calculations based on the Density Functional Theory (DFT) as implemented in the Vienna ab-initio simulation package (VASP). Exchange and correlation effects were incorporated within the generalized gradient approximation (GGA), using the Perdew-Wang 91 (PW91) functional ${ }^{27}$. VoskoWilk-Nusair interpolation ${ }^{28}$ was used for the correlation part of the exchange correlation functional. Interaction between ions and electrons was described using the projector augmented wave method ${ }^{29}$. Cutoff energy was set at $400 \mathrm{eV}$ for the plane-wave basis. In all calculations, self-consistency was achieved with accuracy of at least $10^{-3}$ and $10^{-4} \mathrm{eV}$ for the ionic and electronic loops respectively. All $k$ points were generated using $\Gamma$-centered grids based on the Monkhorst-Pack scheme ${ }^{30}$. The geometry of each periodic supercell has been optimized based on the perfect supercell.

For computational convenience, the primitive $\mathrm{ZnO}$ unit cell was redefined such that for the new basic unit, the a-axis now lies in the original [110]-direction while the b-axis lies in the original [110]-direction. This new basic unit has an orthorhombic crystal lattice that is twice the volume of the primitive hexagonal crystal lattice. The positions of the ions in the crystal are not changed. The pseudoferroelectric domain walls were modeled using a supercell consisting of two identical $180^{\circ}$ (11 10$)$ domain walls. The two $180^{\circ}(1 \overline{1} 0)$ domain walls were separated by 11 bilayers $(31.3 \AA)$. The model was a $62.6 \AA \times 33 \AA \times 5.3 \AA$ supercell and the $k$ points were generated with a $1 \times$ $8 \times 5$ grid. Initial atomic structure at the boundary was modelled after Refs ${ }^{31,32}$, based on what was termed as IDB* (Figs. 4e, 4f). IDB* can be structurally described as a simple IDB (see Supplementary Information, Fig. S9f) that has its inversion domain translated half a unit cell away the [001]-direction. The twin grain boundary, on the other hand, was modeled using a supercell consisting of two identical twin (110) grain boundary. The two (110) twin boundaries are separated by 6 layers of $\mathrm{ZnO}(10.1 \AA)$. This model was a $20.2 \AA \times 3.2 \AA \times 15.9 \AA$ supercell and the $k$ points that were generated with a $1 \times 8 \times 2$ grid. The formation energy of two-dimensional (2D) defects was calculated by using: $E_{F}=1 / 2\left(E-E_{b u l k}\right) / A$, where $E$ is the total energy of the supercell containing two such defects, $\mathrm{E}_{\text {bulk }}$ the total energy of the corresponding perfect supercell with no defects and $A$ is the cross-sectional area of the defect. Bulk $\mathrm{ZnO}$ with and without $\mathrm{V}_{\mathrm{O}}$ was modeled using supercells consisting of $3 \times 3 \times 2$ unit cells, and $k$ points were generated with a $3 \times 3 \times 2$ grid.

In our calculation, the formation energy of a point defect $i$ in neutral state was calculated by using: $E_{f}=E_{\text {tot }}^{d}-E_{\text {tot }}^{0}+\sum n_{i} \mu_{i}$, where $E_{\text {tot }}^{d}$ is the total energy of the supercell with defects, $E_{\text {tot }}^{0}$ the total energy of the corresponding perfect supercell, $n_{i}$ the number of atoms removed, and $\mu_{i}$ the chemical potential of the corresponding atom. The actual chemical potential $\mu_{i}$ depends on experimental growth conditions and ranges from $\mathrm{Zn}$-rich conditions to O-rich conditions. In extreme O-rich condition, $\mu_{O}$ is given by the energy of $\mathrm{O}$ in an $\mathrm{O}_{2}$ molecule while in extreme $\mathrm{Zn}$-rich condition, $\mu_{Z n}$ is given by the energy of $\mathrm{Zn}$ in bulk hcp zinc. In this study, $\mu_{O}$ in O-rich condition was used as the reference and was set as the upper bound of $\mu_{O}$ at $0 \mathrm{eV}$. In addition, for $\mathrm{Zn}$ and $\mathrm{O}$ particle reservoirs to be at thermodynamic equilibrium with bulk $\mathrm{ZnO}$, the following condition has to be satisfied as well: $\mu_{Z n}+\mu_{O}=E_{Z n O}$, where $E_{Z n O}$ is the energy of $\mathrm{ZnO}$. This set the lower bound of $\mu_{O}$ to be at $-3.04 \mathrm{eV}$, which is the heat of formation of $\mathrm{ZnO}$. Our calculated lattice parameters agreed very well with experimental results. For pure $\mathrm{ZnO}$, the computed lattice parameters of $a=b=3.29 \AA, c=5.29 \AA$.

1. Waser, R. \& Aono, M. Nanoionics-based resistive switching memories. Nat Mater 6, 833-840 (2007).

2. Scott, J. F. \& Araujo, C. A. P. D. Ferroelectric Memories. Science 246, 1400-1405 (1989).

3. Lee, S., Kim, H., Yun, D.-J., Rhee, S.-W. \& Yong, K. Resistive switching characteristics of $\mathrm{ZnO}$ thin film grown on stainless steel for flexible nonvolatile memory devices. Appl.Phys. Lett. 95, 262113 (2009).

4. Yang, Y. et al. Observation of conducting filament growth in nanoscale resistive memories. Nat Commun 3, 732 (2012).

5. Joonhyuk, C. et al. Bipolar resistance switching characteristics in a thin $\mathrm{Ti}-\mathrm{Ni}-\mathrm{O}$ compound film. Nanotechnology 20, 175704 (2009).

6. Driscoll, T., Kim, H.-T., Chae, B.-G., Ventra, M. D. \& Basov, D. N. Phasetransition driven memristive system. Appl. Phys. Lett. 95, 043503 (2009).

7. Pershin, Y. V. \& Di Ventra, M. Spin memristive systems: Spin memory effects in semiconductor spintronics. Phys. Rev. B 78, 113309 (2008).

8. Dal Corso, A., Posternak, M., Resta, R. \& Baldereschi, A. Ab initio study of piezoelectricity and spontaneous polarization in ZnO. Phys. Rev. B 50, 10715 (1994).

9. Posternak, M., Baldereschi, A., Catellani, A. \& Resta, R. Ab initio study of the spontaneous polarization of pyroelectric BeO. Phys. Rev. Lett. 64, 1777-1780 (1990).

10. Rodriguez, B. J., Gruverman, A., Kingon, A. I., Nemanich, R. J. \& Ambacher, O. Piezoresponse force microscopy for polarity imaging of GaN. Appl. Phys. Lett. 80, 4166-4168 (2002).

11. Herng, T. S. et al. Mutual Ferromagnetic-Ferroelectric Coupling in Multiferroic Copper-Doped ZnO. Adv. Mater. 23, 1635-1640 (2011).

12. Wang, X. S., Wu, Z. C., Webb, J. F. \& Liu, Z. G. Ferroelectric and dielectric properties of Li-doped $\mathrm{ZnO}$ thin films prepared by pulsed laser deposition. Appl. Phys. A 77, 561-565 (2003).

13. Yang, Y. C. et al. V5+ ionic displacement induced ferroelectric behavior in Vdoped ZnO films. Appl.Phys. Lett. 90, 242903-242901 (2007).

14. Tagantsev, A. K. Pseudoferroelectricity: A possible scenario for doped $\mathrm{ZnO}$. Appl Phys. Lett. 93, 202905-202903 (2008).

15. Park, J. S. et al. Effects of interfacial layer structures on crystal structural properties of ZnO films. J. Vac. Sci. Technol. A 26, 90-96 (2008).

16. Shi, L., Shang, D. S., Sun, J. R. \& Shen, B. G. Bipolar Resistance Switching in Fully Transparent ZnO:Mg-Based Devices. Appl. Phys. Express 2, 101602 (2009).

17. Ozgur, U. et al. A comprehensive review of $\mathrm{ZnO}$ materials and devices. J. Appl. Phys. 98, 041301-041103 (2005).

18. Shi, L. et al. Improved resistance switching in $\mathrm{ZnO}$-based devices decorated with Ag nanoparticles. J. Phys. D: Appl. Phys. 44, 455305 (2011).

19. Tybell, T., Ahn, C. H. \& Triscone, J.-M. Ferroelectricity in thin perovskite films. Appl. Phys. Lett. 75, 856-858 (1999).

20. Jesse, S., Lee, H. N. \& Kalinin, S. V. Quantitative mapping of switching behavior in piezoresponse force microscopy. Rev. Sci. Instrum. 77, 073702 (2006).

21. Sounart, T. L. et al. Sequential nucleation and growth of complex nanostructured films. Adv. Funct. Mater. 16, 335-344 (2006).

22. Sato, Y., Yamamoto, T. \& Ikuhara, Y. Atomic Structures and Electrical Properties of ZnO Grain Boundaries. J. Am. Ceram. Soc. 90, 337-357 (2007).

23. Callister, W. D. \& Rethwisch, D. G. Materials Science and Engineering (Wiley, 2010).

24. Chikazumi, S. \& Charap, S. H. Physics of Magnetism (Krieger Publishing Company, 1978).

25. Seidel, J. et al. Conduction at domain walls in oxide multiferroics. Nat. Mater. 8 , 229-234 (2009)

26. Eliseev, E. A., Morozovska, A. N., Svechnikov, G. S., Gopalan, V. \& Shur, V. Y. Static conductivity of charged domain walls in uniaxial ferroelectric semiconductors. Phys. Rev. B 83, 235313 (2011).

27. Wang, Y. \& Perdew, J. P. Correlation hole of the spin-polarized electron gas, with exact small-wave-vector and high-density scaling. Phys. Rev. B 44, 13298 (1991).

28. Vosko, S. H., Wilk, L. \& Nusair, M. Accurate spin-dependent electron liquid correlation energies for local spin density calculations: a critical analysis. Can. J. Phys. 58, 1200-1211 (1980)

29. Kresse, G. \& Joubert, D. From ultrasoft pseudopotentials to the projector augmented-wave method. Phys. Rev. B 59, 1758 (1999).

30. Monkhorst, H. J. \& Pack, J. D. Special points for Brillouin-zone integrations. Phys. Rev. B 13, 5188 (1976). 
31. Northrup, J. E., Neugebauer, J. \& Romano, L. T. Inversion domain and stacking mismatch boundaries in GaN. Phys. Rev. Lett. 77, 103-106, doi:10.1103/ PhysRevLett.77.103 (1996).

32. Yan, Y. F. \& Al-Jassim, M. M. Inversion domain boundaries in ZnO: Firstprinciples total-energy calculations. Phys. Rev. B 69, 085204 (2004).

\section{Acknowledgements}

The work is supported by NRF-CRP R284-000-056-281 and MoE AcRF R265-000-406-112.

\section{Author contributions}

J.D. and K.Y.Z conceived and designed the experimental and theoretical work. T.S.H. fabricated the ZnO films and performed XRD, Hall Effect analysis, TEM, SEM, EDS, PL, XPS and Raman studies. A.K. carried out the C-AFM, resistance switching, PFM and PFS measurement and A.K performed the corresponding results analysis. C.S.O. developed the model and performed $1^{\text {st }}$ principle calculation. Y.P.F. provided technical guide in modeling/ simulation. T.S.H and C.S.O drafted the manuscript which has been further revised by all authors. T.S.H. and A.K. contributed equally in this work.

\section{Additional information}

Supplementary information accompanies this paper at http://www.nature.com/ scientificreports

Competing financial interests: The authors declare no competing financial interests.

License: This work is licensed under a Creative Commons

Attribution-NonCommercial-NoDerivative Works 3.0 Unported License. To view a copy of this license, visit http://creativecommons.org/licenses/by-nc-nd/3.0/

How to cite this article: Herng, T.S. et al. Investigation of the non-volatile resistance change in noncentrosymmetric compounds. Sci. Rep. 2, 587; DOI:10.1038/srep00587 (2012) 